\title{
Bioimaging with 4th Generation X-Ray Sources
}

\author{
J.B. PEEKA* \\ Institute of Physics, Polish Academy of Sciences, al. Lotników 32/46, 02-668 Warsaw, Poland
}

\begin{abstract}
Newly constructed 4th generation sources of intense synchrotron radiation in ultrafast pulses of only $10-50$ fs and wavelengths up to X-rays, the free electron lasers, are expected to revolutionize development of biological science. To take full advantage of unique properties of the sources, new imaging techniques of molecular and microscopic biological objects are developed. Present article provides a short review of a stormy development of bioimaging with incoming soon 4 th generation synchrotron radiation X-ray sources. Some implications for the future of new sources and techniques are discussed as well.
\end{abstract}

PACS numbers: 87.59.-e, 41.60.Cr, 07.85.Qe, 82.53.Ps, 87.64.Bx

\section{Introduction}

Since few decades, synchrotron radiation (SR) has constituted an excellent tool for techniques probing lifeforms, from single molecules, subcellular structures, to cells, tissues and organisms. X-ray SR sources revolutionized biological imaging. Several techniques exploiting phase contrast $(\mathrm{PC})$, including X-ray interferometry, diffraction-enhanced imaging, and in-line PC radiography benefit particularly well of characteristic properties of synchrotron beams [1]. Phase contrast, due to its sensitivity, up to $10^{3}$ times higher in the range of photon energies between 15 and $35 \mathrm{keV}$, as compared to absorption contrast, can be monitored by SR even in cases, when absorption differences are extremely small [2-4]. $\mathrm{SR}$ is also widely used to determination of biologically active molecular structures [5].

Various techniques of bioimaging have been applied at virtually all of about 80 synchrotron facilities operating over the world. Beamtime allotted to biomedical applications cover today about $40 \%$ of total beamtime at many $\mathrm{SR}$ facilities. This is more than applications in material research, which dominated still few years ago.

A risk of damage is particularly important factor in probing the biostructures and dynamics of life processes in organisms or their parts. This is the radiation damage, that imposes stringent constraints to irradiation procedures and introduces ultimate limitations to classical imaging techniques with X-rays. Conventional methods cannot achieve atomic resolution on non-repetitive structures due to damage that develops during the experiment. Even in case of tissue preparations, the biodegradation due to irradiation may substantially affect results.

A compromise between acceptable irradiation dose and spatial or temporal resolution of imaging, insufficient dose rates for some dynamic studies of lifeforms, or specimen preparation techniques resulting in artifacts, en-

* e-mail: pelkay@ifpan.edu.pl tail characteristic limitations on application of traditional synchrotron sources to biomedical research. To the most common examples of these types of limitations counts protein imaging, that will be discussed later in this work. A breakthrough in overcoming the restriction is expected at extreme dose rates and ultrashort exposures with construction of new 4th generation SR sources (4GLS), the free electron lasers (FELs) [6].

The present article is thought as a snapshot, which provides, for obvious reasons fragmented and incomplete, view of a stormy development of bioimaging with incoming soon 4th generation of X-ray sources. Some implications for the future will be discussed as well.

\section{Imaging with 4 th generation $\mathrm{SR}$ sources}

FELs utilize a relativistic electron beam as a lasing medium. As a result, a monochromatic radiation is generated in ultrafast pulses, of duration down to the fs range, with a unique combination of tunability, coherence, polarization, and high power that can exceed more than $1 \mathrm{GW}$ in a single pulse. Some FEL types deliver radiation in the spectral regions of short wavelengths, ranging from vacuum ultraviolet (VUV-FEL) to X-rays (X-FEL), particularly suitable to a fine imaging. Spectral brightness of the new X-FEL sources, to be operational in the next few years, will be up to 9 orders of magnitude higher, as compared to the most intense 3GLS sources, reaching a value of $10^{29}$ photons/ ( $\mathrm{s} \mathrm{mrad}^{2} \mathrm{~mm}^{2} 0.1 \% \mathrm{BW}$ ), emitted in ultrafast pulses of only $10-20 \mathrm{fs}$ [7]. Since a decade operational are FEL sources delivering XUV radiation.

A decisive impact on biological science and related technology is expected with new techniques of imaging possible only with these sources. In particular, X-FELs will certainly revolutionize structural biology with a unique opportunity of a variant of the coherent X-ray diffraction imaging (CXDI) applicable to nonperiodic structures, from single molecules to cells and tissue preparations, at a spatial resolution in subnanometer 
to micrometer scales, especially important in biological studies $[8,9]$.

Coherent X-ray diffraction imaging was firstly demonstrated by Miao in 1999 [10], who extended to X-ray region earlier experiments on image reconstruction from diffraction pattern in visible light [11]. First CXDI result of Escherichia coli bacteria stained with $\mathrm{KMnO}_{4}$, obtained with radiation of $\lambda=0.2 \mathrm{~nm}$ at resolution of $30 \mathrm{~nm}$ was reported in 2003 [12].

\section{Imaging proteins with 3rd and 4th generation $\mathrm{X}$-ray sources}

\subsection{Protein structure investigation with 3rd generation $S R$ sources}

Synchrotron radiation is widely used to determination of biologically active molecular structures [13]. More than $80 \%$ protein structures, out of 50000 collected in Protein Data Bank (PDB), have been solved with aid of $\mathrm{X}$-ray beams delivered by, mostly, 3GLS dedicated synchrotron storage rings. This structural expertise contributes significantly in better understanding of fundamental life processes and in exploring interaction of drugs with organisms and with pathogens. It helps in advancing the design of drugs, enabling greatly reduced dosages and improved outcomes. However, these 50000 proteins with solved structures, are only about $6 \%$ of all proteins $(\approx 800000)$ sequenced up to now. In this, less than $500(<0.1 \%)$ are membrane proteins (MP) with solved structures [14]. On the other hand, $70 \%$ of today's drugs are aimed at MPs.

Classical diffraction approach to structure determination with SR is loaded with impassable limits. To proceed with structure analysis a crystal is necessary. Unfortunately, about $40 \%$ proteins cannot be crystallized with any of known methods, therefore conventional crystallography is not applicable in this case. Moreover, even crystalline proteins have frequently modified conformation, by packing, and by unnatural environment that can lead to misinterpretation. Among these extremely difficult to crystallize and purify, are just almost all MPs. Other example can be unsoluble peptide fibrils of beta-amyloid, that take part in Alzheimer's disease.

We need new methods of non-crystalline protein structure investigation to push forward challenging game with diseases, and to get insight in the living processes as close as possible.

\subsection{4 th generation sources. New approach to bioimaging}

Theory predicts, that with an ultrafast, intense and coherent X-ray pulse a diffraction pattern can be recorded from a single object (1-1000 nm), like a molecule, a virus or a cell before the object is damaged and converted into plasma [6]. This makes use of quite new philosophy: Do not care about damage. Measure before it occurs. The new approach is possible only with extremely intense coherent sources, like X-ray FEL, since a combination of extreme photon flux, coherence and the ultrafast pulse should be fulfilled at the same time, together with excellent spatial stability and repeatability of the source. This new technique is in fact a form of CXDI having developed at ordinary SR sources since more than a decade. With the new technique slightly disturbing is that we have either the object or information about its structure. Be cautious to study valuable unique objects this way.

To apply the CXDI with atomic resolution, an intense monochromatic and highly coherent pulsed source is necessary, which operates at a wavelength of about $0.1 \mathrm{~nm}$ with pulses of duration below $50 \mathrm{fs}$, containing $10^{12}$ photons each. The whole energy of the pulses should be focused on the investigated specimen. It means, in case of single protein diffraction, a need to focus the beam to 10 $100 \mathrm{~nm}$. Real image of an object is derived from recorded intensity distribution of the diffracted beam with phase retrieval algorithm.

To determine the $3 \mathrm{D}$ structure of a protein, a multishot experiment is necessary with $>10^{5}$ shots, each interacting with a new molecule. To achieve this, a molecular injector is applied that forms a train of protein molecules, precisely synchronized with FEL pulses exactly in the beam focus.

Free electron lasers based on self-amplified spontaneous emission (SASE) effect can deliver tunable, highly coherent monochromatic radiation in the XUV to X-ray range of wavelengths in ultrashort pulses of only 1050 fs and of peak power exceeding 1 GW [15]. SASE-FELs have been recognized as excellent sources to probe the dynamics of ultrafast processes and the structure of matter with unprecedented spatial and temporal resolution. This makes it possible to apply methods inaccessible with other known types of radiation sources. After the XUV-FLASH, the prototype of SASE-FEL facility constructed in HASYLAB (Hamburg) has passed successfully examination, confirming thus theoretical expectations, the construction of next three devices working in the range of hard X-rays started. Firstly to be operated is LCLS at SLAC, USA (in 2009), next RIKEN XFEL at Harima, Japan (2010?), and finally EU-XFEL at DESY, Germany (2013). The project of the European X-FEL is the most advanced; the facility is constructed and will be exploited by international consortium in which Poland also participates [16].

\section{Testing new sources and new techniques with XUV-FEL}

Power and flexibility of short-wavelength FELs are just being extensively studied with the FLASH facility in DESY (Hamburg), operating in the XUV photon energy range. Test results show that FELs radiating in the range of XUV to X-rays will offer unparalleled opportunities for the study of processes occurring on ultrashort time and length scales, in medicine, biology, biochemistry, and in related fields of science and technology [17]. 
Before X-FEL will start, a number of crucial issues related to the bioimaging experiments should be solved. Among them are improvements of phase retrieval algorithms and classification schemes necessary to calculate the $3 \mathrm{D}$ molecule structure from the set of more than $10^{5}$ single shot diffraction patterns. Construction of injectors capable to deliver precisely a train of molecules with their natural structures conserved, as well as construction of ultrafast, low-noise detectors to record diffracted single-photon counts from a $10^{12}$ photon pulse are other examples of technical tasks to be solved. And more, more other...

To clarify are also more fundamental obstacles. Recently, a question of a limit to a molecule size, which can be analyzed has been discussed by Hau-Riege [18]. He noticed that maximal size of about $300 \mathrm{kDa}$ can be anticipated for the 3D image reconstruction of a molecule due to structure of pulses produced in SASE-FEL process. Each pulse is composed of statistically uncorrelated narrow spikes, that constrains the temporal coherence of the X-FEL radiation to the order of 300 as.

A number of results on CXDI imaging with XUVFLASH of microscopic objects has confirmed theoretical fundamentals. Among first experiments was a single pulse diffraction from Spiroplasma cells. Spiroplasma are small procaryotic bacteria (class Mollicutes), with long $(>1 \mu \mathrm{m})$ and thin $(\approx 100 \mathrm{~nm})$ cells, that infect plants. Single shot diffraction has been recorded at the wavelength of $13.5 \mathrm{~nm}$. Electron density distribution of the bacteria calculated with a complex phase-retrieval algorithm was directly comparable with scanning electron microscopy (SEM) images, recorded prior to FEL exposition $[19,20]$. Another experiment was performed with a micrometer-scale pattern etched in a thin amorphous SiN membrane with radiation at wavelength of $32 \mathrm{~nm}$ [21]. Reconstruction was excellent with calculated resolution of $62 \mathrm{~nm}$.

Since the very start of the XUV-FLASH in 1999, a group of Polish scientists joined an international team aimed on experimental study of damage induced by intense ultrafast FEL pulses in solids. Some of the experiments were the first that supported one of fundamental theoretical assumptions of CXDI with damaging pulses. Basing on studies of spacing parameter of specially designed multilayers exposed to XUV FEL pulses and probing dynamics of damage in ultrathin membranes by a single-color pump-and-probe technique, it was confirmed that the damage processes of solids are effectively delayed of about 25 fs after pulse starts $[17,22]$.

\section{Final remarks - future of the SR $\mathrm{X}$-ray sources}

SASE-FEL is a large-scale facility composed of a linear electron accelerator, a long undulator and bunch compressors ("chicanes") as its main parts. To initiate the SASE process in undulator, the electron beam, divided to a train of ultrashort pulses of possibly highest electron density, should be accelerated to few hundreds MeVfew $\mathrm{GeV}$. This is achieved with a superconducting RF linear accelerator operating at an electric field that cannot exceed, typically, the intensity of about $30 \mathrm{MV} / \mathrm{m}$ in the presently constructed linacs. With this $E$-field, the accelerator should be as long as few hundred meters to produce electron bunches of sufficient energy. This makes the FEL facility very large and expensive.

It is clear that if new bioimaging techniques with X-FELs, especially the CXDI, worked, as it can be safely anticipated from results of up-to-date study described above, we would urgently need compact, intense sources with parameters similar to X-FEL. Some candidates are here sources based on the inverse Compton scattering (an operating example at Vanderbildt University), plasma sources, HGHG generators, etc.

Recently, Jaroszynski et al. [23] reported on demonstration of the first successful combination of a laser-plasma wakefield accelerator, producing $55-75 \mathrm{MeV}$ electron bunches, with an undulator to generate visible synchrotron radiation. With the light focused from a $5 \mathrm{TW}$ optical laser pulse into a $2 \mathrm{~mm}$ wide gas jet, a beam of electrons with a peak energy of 55-75 MeV has been obtained as a result of interaction of the laser with the jet. The beam has been directed into a $1 \mathrm{~m}$ long undulator producing light at the red end of the visible spectrum (with the wavelength of about $950 \mathrm{~nm}$ ). As it is noted by authors, this approach, after some improvements, could substantially reduce both the size and cost of synchrotron and FEL X-ray sources, through the use of electron beams produced by a laser-driven particle accelerator.

The laser-plasma wakefield accelerator uses the immense electric fields produced in the plasma at the focus of ultrahigh intensity lasers to accelerate electrons over distances of just centimeters - thousands of times shorter than a conventional particle accelerator. The relative energy spread can be soon minimized to the order of $0.1 \%$ for a $1 \mathrm{GeV}$ beam, with an emittance down to $0.1-1 \pi \mathrm{mm}$ mrad. With these parameters an electron bunch of length as short as $10 \mathrm{fs}$, and an effective beam current of up to $100 \mathrm{kA}$ could be produced without the need for a compression stage by chicanes. This would substantially reduce the required undulator length to just a few meters, instead of tens to few hundred meters, that are necessary in SASE-FEL [24].

\section{Acknowledgments}

This work was partially supported by the grant of Ministry of Science and Higher Education, SPB no. DESY $/ 68 / 2007$.

\section{References}

[1] R. Meuli, Y. Hwu, J.H. Je, G. Margaritondo, Eur. Radiol. 14, 1550 (2004).

[2] A. Bravin, J. Phys. D, Appl. Phys. 36, A24 (2003). 
[3] T. Takeda, Nuclear Instrum. Methods Phys. Res. A 548, 38 (2005).

[4] J.B. Pełka, Acta Phys. Pol. A 114, 309 (2008).

[5] T.L.-M. Sorensen, K.E. McAuley, R. Flaig, E.M.H. Duke, Trends Biotechnol. 24, 500 (2006).

[6] R. Neutze, R. Wouts, D. van der Spoel, E. Weckert, J. Hajdu, Nature 406, 752 (2000).

[7] N. Patel, Nature 415, 110 (2002).

[8] R. Neutze, G. Huldt, J. Hajdu, D. van der Spoel, Radiat. Phys. Chem. 71, 905 (2004).

[9] H.N. Chapman, A. Barty, M.J. Bogan, S. Boutet, M Frank, S.P. Hau-Riege, S. Marchesini, B.W. Woods, S. Bajt, W.H. Benner, R.A. London, E. Plonjes, M. Kuhlmann, R. Treusch, S. Dusterer, T. Tschentscher, J.R. Schneider, E. Spiller, T. Möller, C. Bostedt, M. Hoener, D.A. Shapiro, K. O. Hodgson, D. van der Spoel, F. Burmeister, M. Bergh, C. Caleman, G. Huldt, M.M. Seibert, F.R.N.C. Maia, R.W. Lee, A. Szoke, N. Timneanu, J. Hajdu, Nature Phys. 2, 839 (2006).

[10] J. Miao, P. Charalambous, J. Kirz, D. Sayre, Nature 400, 342 (1999).

[11] J.R. Fienup, J. Opt. Soc. Am. A 4, 118 (1987).

[12] J. Miao, K.O. Hodgson, T. Ishikawa, C.A. Larabell, M.A. LeGros, Y. Nishino, Proc. Natl. Acad. Sci. 100, 110 (2003).

[13] T.L.-M. Sorensen, K.E. McAuley, R. Flaig, E.M.H. Duke, Trends Biotechnol. 24, 500 (2006).

[14] Protein Data Bank, PDB, August 2008.

[15] R. Bonifacio, C. Pellegrini, L.M. Narduci, Opt. Commun. 50, 373 (1984).

[16] http://www.xfel.eu/en/.

[17] S.P. Hau-Riege, H.N. Chapman, J. Krzywinski, R. Sobierajski, S. Bajt, R.A. London, M. Bergh, C. Caleman, R. Nietubyc, L. Juha, J. Kuba, E. Spiller, S. Baker, R. Bionta, K. Sokolowski Tinten, N. Stojanovic, B. Kjornrattanawanich, E. Gullikson, E. Plönjes, S. Toleikis, T. Tschentscher, Phys. Rev. Lett. 98, 145502 (2007).

[18] S.P. Hau-Riege, Opt. Express 16, 2840 (2008).
[19] H. Chapman, J. Hajdu, I. Andersson, S. Bajt, A. Barty, W.H. Benner, M. Bergh, M.J. Bogan, C. Bostedt, S. Boutet, F. Burmeister, C. Caleman, S. Düsterer, T. Ekeberg, M. Frank, S.P. Hau-Riege, M. Hoener, B. Iwan, M. Kuhlmann, R.A. London, F.R.N.C. Maia, S. Marchesini, E. Marklund, Th. Möller, D. DePonte, U. Rohner, A.E. Sakdinawat, M.M. Seibert, D.A. Shapiro, E. Spiller, M. Svenda, A. Szoke, N. Timneanu, R. Treusch, U. Weierstall, B.W. Woods, Hasylab Reports 2007, 437 (2008).

[20] Schaevitz, Lee, and Fletcher, Cell 122, 941 (2005).

[21] H.N. Chapman, J. Hajdu, S. Bajt, A. Barty, D. Barsky, W.H. Benner, M. Bergh, M.J. Bogan, C. Bostedt, S. Boutet, C. Caleman, F. Burmeister, S. Düsterer, M. Frank, S.P. Hau-Riege, K.O. Hodgson, M. Hoener, G. Huldt, M. Kuhlmann, R.W. Lee, R.A. London, F.R.N.C. Maia, S. Marchesini, E. Marklund, Th. Möller, E. Plönjes, U. Rohner, J.R. Schneider, M.M. Seibert, D.A. Shapiro, E. Spiller, D. van der Spoel, A. Szoke, N. Timneanu, R. Treusch, Th. Tschentscher, B.W. Woods, Hasylab Reports 2007, 423 (2007).

[22] S.P. Hau-Riege, R.A. London, R.M. Bionta, M.A. McKernan, S.L. Baker, J. Krzywinski, R. Sobierajski, R. Nietubyc, J.B. Pelka, M. Jurek, L. Juha, J. Chalupský, J. Cihelka, V. Hájková, A. Velyhan, J. Krása, J. Kuba, H. Wabnitz, K. Tiedtke, S. Toleikis, T. Tschentscher, M. Bergh, C. Caleman, K. Sokolowski Tinten, N. Stojanovic, U. Zastrau, Appl. Phys. Lett. 90, 173128 (2007).

[23] H.-P. Schlenvoigt, K. Haupt, A. Debus, F. Budde, O. Jäckel, S. Pfotenhauer, H. Schwoerer, E. Rohwer, J.G. Gallacher, E. Brunetti, R.P. Shanks, S. M. Wiggins, D.A. Jaroszynski, Nature Phys. 4, 130 (2008).

[24] K. Nakajima, Nature Phys. 4, 92 (2008). 